\title{
Structural Reliability Based Dynamic Positioning of Turret-Moored FPSOs in Extreme Seas
}

\author{
Yuanhui Wang, Chuntai Zou, Fuguang Ding, Xianghui Dou, Yanqin Ma, and Yanwen Liu \\ College of Automation, Harbin Engineering University, Harbin 150001, China \\ Correspondence should be addressed to Fuguang Ding; dingfuguang@hrbeu.edu.cn
}

Received 12 February 2014; Revised 9 June 2014; Accepted 10 June 2014; Published 7 July 2014

Academic Editor: Oleg V. Gendelman

Copyright (C) 2014 Yuanhui Wang et al. This is an open access article distributed under the Creative Commons Attribution License, which permits unrestricted use, distribution, and reproduction in any medium, provided the original work is properly cited.

FPSO is widely used during the deep-sea oil and gas exploration operations, for which it is an effective way to keep their position by means of positioning mooring (PM) technology to ensure the long-term reliability of operations, even in extreme seas. Here, a kind of dynamic positioning (DP) controller in terms of structural reliability is presented for the single-point turret-moored FPSOs. Firstly, the mathematical model of the moored FPSO in terms of kinematics and dynamics is established. Secondly, the catenary method is applied to analyze the mooring line dynamics, and mathematical model of one single mooring line is set up based on the catenary equation. Thereafter, mathematical model for the whole turret mooring system is established. Thirdly, a structural reliability index is defined to evaluate the breaking strength of each mooring line. At the same time, control constraints are also considered to design a state feedback controller using the backstepping technique. Finally, a series of simulation tests are carried out for a certain turret-moored FPSO with eight mooring lines. It is shown in the simulation results that the moored FPSO can keep its position well in extreme seas. Besides, the FPSO mooring line tension is reduced effectively to ensure mooring lines safety to a large extent in harsh sea environment.

\section{Introduction}

Floating production storage and offloading vessels (FPSOs) are vessels or platforms that float in deep water. The offshore oil and gas industry uses them to process crude and natural gas after it is pumped up from deep beneath the ocean floor. They may also assist in the pumping process itself. Once a new oilfield is discovered and numerous production wells are drilled, an FSPO is put in place and the various platforms and underwater wellheads are connected to it.

With the continued global thirst for oil and natural gas, deep-sea oil and gas explorations have become imperative and challengeable. PM systems, also, namely, thruster assisted position mooring systems, have been proved to be a more cost-efficient alternative to floating offshore structures such as FPSOs and semisubmersibles. PM systems combine traditional mooring system with modern dynamic position system to acquire both their advantages. In normal sea conditions, the mooring system constrains the position of the vessel, while the dynamic positioning controller calculates out the thruster forces to regulate the heading, only. However, in harsh seas, thrusters must assist the mooring system altogether to keep positioning well.

Strand et al. [1] proposed that automatic heading setpoint control is the most important function for turret-anchored ships. Nguyen and Sørensen [2] proposed a new concept of setpoint chasing for moderate and extreme sea conditions. Nguyen and Sørensen [3] designed a switching control method for PM systems, which allows the thrusters to assist the mooring system in varying environmental and vessel operational conditions. Berntsen et al. $[4,5]$ developed a reliability based control algorithm with an intrinsic part of reliability index. Fang and Blanke [6] introduced a novel way to determine the reference position to protect mooring lines and Fang et al. [7] extended the former results by using the structural reliability index to calculate optimal setpoint. However, the formerly proposed structural reliability index by Berntsen et al. [4] is equal to the critical reliability index, which cannot make sure that all the mooring lines are not broken down in extreme seas.

Here, a dynamic positioning controller in terms of structural reliability is presented for moored FPSOs to 


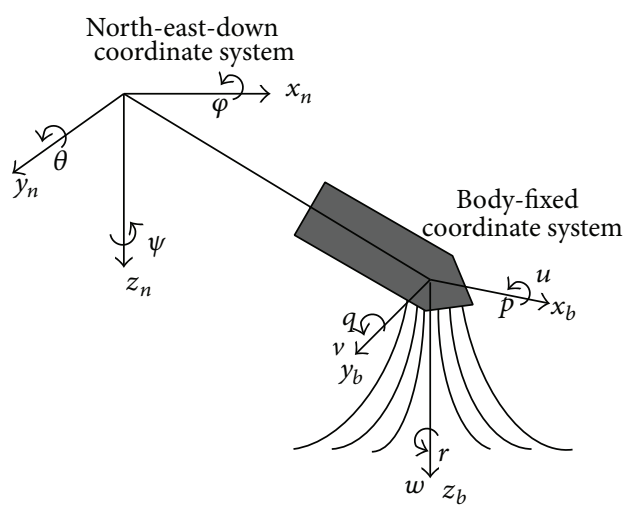

FIGURE 1: Reference coordinate system.

protect the mooring lines from breakage in extreme seas, in which the structural reliability index is selected a little larger than the critical reliability index. First of all, the kinematics and dynamics mathematical models of FPSOs are established. Then the mooring system models are also established. Thereafter, a control algorithm of PM system based on structural reliability is proposed. Finally, a number of simulation experiments are carried out for a certain singlepoint turret-moored FPSO in extreme seas, concerned more with the safety of mooring lines.

\section{Mathematical Model of Turret-Moored FPSOs}

2.1. FPSOs Kinematics. The north-east-down coordinate system is denoted as inertial frame, in which the motions of vessel are described as shown in Figure $1 .\left(x_{b}, y_{b}, z_{b}\right)$ is the position of the center of vessel in north-east-down coordinate system; $(\varphi, \theta, \psi)$ represents the attitude angle of vessel in north-east-down coordinate system; $\varphi$ is rotated around the $x$-axis named roll; $\theta$ is rotated around the $y$-axis named pitch; $\psi$ is rotated around the $z$-axis named yaw; and $u, v, w, p, q, r$ are the transitional and rotational velocities in body-fixed coordinate system.

For simplicity, only the three-degree-of-freedom motions of the vessels in the horizontal plane (surge, sway, and yaw) are considered for the moored FPSOs. Then, the nonlinear low-frequency model is established as follows [8]:

$$
\begin{gathered}
M \dot{\nu}+C_{\mathrm{RB}}(\nu) \nu+C_{A}\left(v_{r}\right) v_{r}+D_{L} \nu+D_{\mathrm{NL}}\left(\nu_{r}, \gamma_{r}\right) v_{r} \\
=\tau_{\mathrm{th}}+\tau_{w}+\tau_{m}, \\
\dot{\eta}=J(\eta) v,
\end{gathered}
$$

where $M$ is the inertia matrix including added mass, $C_{\mathrm{RB}}(\nu)$ and $C_{A}\left(\nu_{r}\right)$ are the skew-symmetric Coriolis and centripetal matrices of the rigid body and added mass, $D_{L}$ is a strictly positive damping matrix, caused by linear wave drift damping and laminar skin friction, and $D_{\mathrm{NL}}\left(\nu_{r}, \gamma_{r}\right)$ is a nonlinear damping matrix. $\tau_{w}$ is the environmental load vector; $\tau_{\text {th }}$ is a vector of control force provided by the thruster system; $\tau_{m}$ is the mooring force vector; $v$ is the velocity matrix in

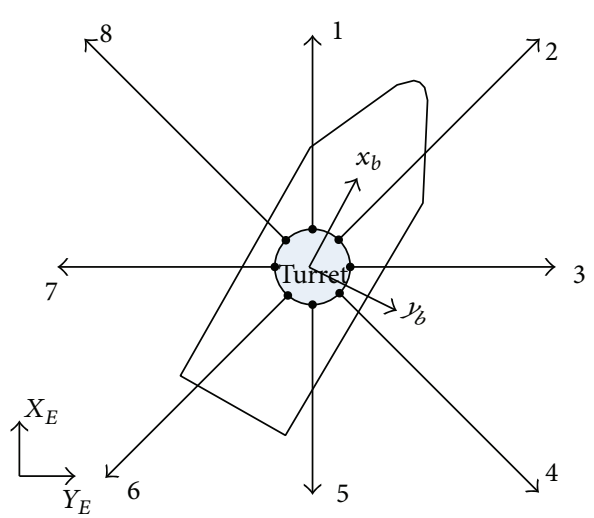

Figure 2: The arrangement of mooring lines.

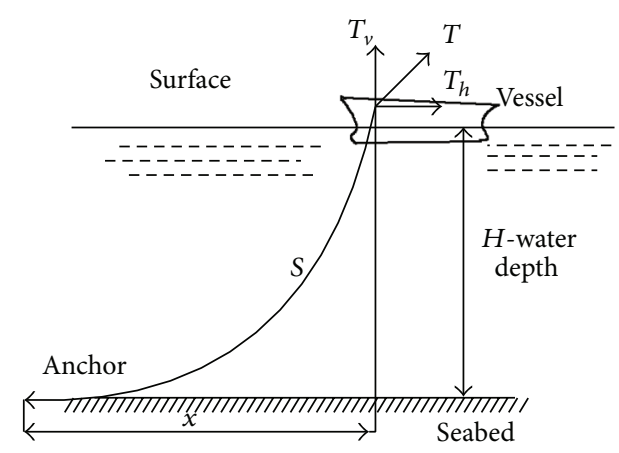

Figure 3: Schematic diagram of a single mooring line.

body-fixed coordinate; $\eta$ is the position matrix of vessel; $J(\eta)$ is a rotation matrix.

2.2. Mooring System Model. Here, the mooring system of an internal turret-moored FPSO is comprised of eight mooring lines as an example, and the mooring lines are connected to the vessel through the turret and fixed to the seabed by means of anchors as shown in Figure 2. Each mooring line is in the general form of a catenary chain line as shown in Figure 3. The forces on the mooring lines are analyzed by catenary theory as follows [9]:

$$
s-X=h \sqrt{1+\frac{2 T_{h}}{\omega h}}-\frac{T_{h}}{\omega} \cosh ^{-1}\left(1+\frac{\omega h}{T_{h}}\right),
$$

where $s$ is the length of the mooring line; $X$ is the length in the horizontal projection of the mooring line; $h$ is the water depth; $T_{h}$ is the horizontal tension of mooring line acting on the turret; $\omega$ is the unit weight of mooring line in water.

Assuming the mooring lines of internal turret-moored FPSO are symmetrically located, the initial length of each line is $s_{i}$, the position of the anchor fixed is $\left(x_{i}, y_{i}\right), i=1, \ldots, 8$, and the position of the turret center is $\left(x_{0}, y_{0}\right)$. All start points 
of the lines from the turret are considered to be the same one, and the following equation can be derived as follows [9]:

$$
\begin{gathered}
X_{i}=\sqrt{\left(x_{i}-x_{0}\right)^{2}}+\sqrt{\left(y_{i}-y_{0}\right)^{2}}, \\
s_{i}-X_{i}=h \sqrt{1+\frac{2 T_{h i}}{\omega h}}-\frac{T_{h i}}{\omega} \cosh ^{-1}\left(1+\frac{\omega h}{T_{h i}}\right), \\
\theta_{i}=\arctan \left(\frac{y_{i}-y_{0}}{x_{i}-x_{0}}\right), \\
i=1, \ldots, 8,
\end{gathered}
$$

where $\theta_{i}$ is the azimuth angle of the $i$ th mooring line and $T_{h i}$ is the horizontal tension of the $i$ th mooring line acting on the turret. Thus,

$$
\begin{gathered}
X_{h i}=T_{h i} \cos \left(\theta_{i}\right), \quad Y_{h i}=T_{h i} \sin \left(\theta_{i}\right), \\
N_{h i}=0, \quad X_{\text {all }}=\sum_{i=1}^{8} X_{h i}, \\
Y_{\text {all }}=\sum_{i=1}^{8} Y_{h i}, \quad N_{\text {all }}=0,
\end{gathered}
$$

where $X_{\text {all }}, Y_{\text {all }}$, and $N_{\text {all }}$ denote the sum of all mooring forces in related coordinate axis.

Finally, the forces calculated in NED coordinate system are transformed to the body-fixed coordinate system as follows:

$$
\tau_{m}=\left[\begin{array}{c}
X_{m} \\
Y_{m} \\
N_{m}
\end{array}\right]=J^{T}(\psi)\left[\begin{array}{c}
X_{\text {all }} \\
Y_{\text {all }} \\
N_{\text {all }}
\end{array}\right]
$$

\section{Dynamic Positioning Controllers Considering Structural Reliability for Moored FPSOs}

3.1. Structural Reliability Index. The main objective of the controllers design is to ensure that the FPSOs are well positioned as well as ensuring that the moored lines are not overloaded in extreme seas. So it is important to quantify the load of each mooring line with respect to its expected endurance. Here, a structural reliability index is introduced to quantify the probability of mooring line failure, and it is expressed as [10]

$$
\delta_{k}(t)=\frac{T_{b, k}-k_{k,} \sigma_{k}-T_{k}(t)}{\sigma_{b, k}}, \quad k=1, \ldots, q,
$$

where $T_{b, k}$ is the mean breaking strength of mooring line $k ; \sigma_{k}$ is the standard deviation of the time varying tension (including high frequencies); $k_{k}$ is a scaling factor; $T_{k}(t)$ is the low frequency part of the mooring line tension; $\sigma_{b, k}$ is the standard deviation of the mean breaking strength. A lower bound is selected for $\delta_{k}$; $\delta_{s}$ defines the critical value of the reliability index. The condition $\delta_{k}<\delta_{s}$ represents a situation where the probability of line failure is intolerably high.
Formula (6) describes the reliability criterion for line $k$. The controllers will act based on the most critical reliability index, so the subscript $j$ is used to identify the smallest reliability index:

$$
\delta_{j}(t)=\min _{k \in\{1, \ldots, q\}} \delta_{k}(t),
$$

where $q$ represents the number of mooring lines.

3.2. Controllers Design Based on Mooring Line Structural Reliability. Feedback backstepping controllers are proposed to prevent not only the collision between FPSOs and shuttle tankers, but also the breakage of mooring lines in extreme seas.

The following assumptions on the mooring system are proposed.

Assumption 1. The two constants $\varepsilon_{1}$ and $\varepsilon_{2}$ satisfy

$$
0<\varepsilon_{1} \leq T_{j}^{\prime} \leq \varepsilon_{2},
$$

for all $t \in \mathfrak{R}_{+}$.

Assumption 2. There is a constant $\rho$ such that

$$
r_{j} \geq \rho>0,
$$

where $r_{j}$ denotes the extension of mooring line $j$, and the time derivative of $\delta_{j}$ is as follows:

$$
\dot{\delta}_{j}(t)=-\frac{\dot{T}_{j}(t)}{\sigma_{b, j}}
$$

and since

$$
\dot{T}_{j}(t)=T_{j}^{\prime} \dot{r}_{j}=\frac{T_{j}^{\prime}}{r_{j}}\left(p-p_{j}\right)^{T} \dot{p},
$$

then

$$
\dot{\delta}_{j}(t)=-\frac{T_{j}^{\prime}}{\sigma_{b, j} r_{j}}\left(p-p_{j}\right)^{T} J_{2}(\psi) w .
$$

State feedback controllers are proposed that regulate $\left(\nu, \psi, \delta_{j}\right)$ to $\left(0, \psi_{d}, \delta_{d}\right), \psi_{d}, \delta_{d}$ are the desired yaw angle and desired minimum reliability index. It is quite different from the idea of Berntsen et al. [4] that the value of $\delta_{d}$ is selected larger than the critical reliability index to ensure that $\delta_{j}>\delta_{s}$. Here, $\delta_{d}>\delta_{s}$. Thus, even in extreme seas, the mooring lines will still not be broken down with strong effects from the harsh wind gust.

The state feedback backstepping controllers have been developed and the propositions are presented in [11-13]. Here, $\eta=\left[P^{T}, \psi\right]^{T}=[x, y, \psi]^{T}$ is the position and heading in north-east-down coordinate system; $v=\left[w^{T}, \rho\right]^{T}=[u, v, \rho]^{T}$ is the transitional and rotational velocities in body-fixed coordinate system. $\tau_{\mathrm{BSP}}$ is the output force and moment of the state feedback controllers. 
Proposition 3. $\lambda, \gamma$, and $\kappa$ are strictly positive constants. Then, the control law is as follows:

$$
\tau_{B S P}=M \varsigma+D v+g(\eta)-J^{T}(\psi) b,
$$

where

$$
\varsigma=\left[\begin{array}{c}
\left.\left(-\lambda+\frac{\gamma}{r_{j}}\left(\delta_{j}-\delta_{d}\right)\right)\right]\left[\begin{array}{c}
w \\
\rho
\end{array}\right] \\
-(\lambda+\kappa)
\end{array}\right]+\left[\begin{array}{c}
\left(\begin{array}{c}
-\left(\frac{T_{j}^{\prime} \gamma}{\sigma_{b, j}}+\frac{\gamma}{r_{j}}\left(\delta_{j}-\delta_{d}\right)\right) \\
\varsigma
\end{array}\right) \vartheta^{T} w-\kappa \gamma\left(\psi-\psi_{d}\right) \\
+\left(\lambda \gamma+\frac{T_{j}^{\prime}}{\sigma_{b, j}}\right)\left(\delta_{j}-\delta_{d}\right) I_{2} \\
\left.+\left(\delta_{j}-\delta_{d}\right) \gamma \rho S_{2}\right) \vartheta \\
\vartheta
\end{array}\right.
$$

renders the solution $\left(\nu, \delta_{j}, \psi\right)=\left(0, \delta_{d}, \psi_{d}\right)$ of $(1)$ and (15) to be globally exponentially stable (GES).

Proof. Consider the following Lyapunov function candidate:

$$
V_{1}=\frac{1}{2}\left(\delta_{j}-\delta_{d}\right)^{2}+\frac{1}{2}\left(\psi-\psi_{d}\right)^{2} .
$$

Its time derivative is

$$
\begin{aligned}
\dot{V}_{1} & =\left(\delta_{j}-\delta_{d}\right) \dot{\delta}_{j}+\left(\psi-\psi_{d}\right) \dot{\psi} \\
& =-\frac{T_{j}^{\prime}(t)}{\sigma_{b} r_{j}(t)}\left(\delta_{j}-\delta_{d}\right)\left(p-p_{j}\right)^{T} J_{2}(\psi) w+\left(\psi-\psi_{d}\right) \rho .
\end{aligned}
$$

Choosing $w$ and $\rho$ as virtual inputs that

$$
\begin{aligned}
& w=\alpha_{w} \stackrel{\Delta}{=} r_{j}\left(\delta_{j}-\delta_{d}\right) \gamma J_{2}^{T}(\psi)\left(p-p_{j}\right), \\
& \rho=\alpha_{\rho} \stackrel{\Delta}{=}-\kappa\left(\psi-\psi_{d}\right)
\end{aligned}
$$

exponentially stabilizes $\left(\delta_{j}, \psi\right)=\left(\delta_{d}, \psi_{d}\right)$, since (17) substituted into (16) yields

$$
\dot{V}_{1} \leq-\frac{\varepsilon_{1} \gamma}{\sigma_{b}}\left(\delta_{j}-\delta_{d}\right)^{2}-\kappa\left(\psi-\psi_{d}\right)^{2}
$$

Defining the change of variables

$$
z=\left[\begin{array}{c}
z_{w} \\
z_{\rho}
\end{array}\right] \stackrel{\Delta}{=} v-\alpha
$$

where $\alpha=\left[\alpha_{w}^{T}, \alpha_{\rho}\right]^{T}$, and augmenting Lyapunov function to obtain

$$
V_{2}=V_{1}+\frac{1}{2} z^{T} z
$$

get

$$
\dot{V}_{2} \leq-\frac{\varepsilon_{1} \gamma}{\sigma_{b, j}}\left(\delta_{j}-\delta_{d}\right)^{2}-\kappa\left(\psi-\psi_{d}\right)^{2}+z^{T}(\theta+\dot{z}),
$$

where

$$
\theta=\left[-\frac{T_{j}^{\prime}}{\sigma_{b, j} r_{j}}\left(\delta_{j}-\delta_{d}\right) J_{2}^{T}(\psi)\left(p-p_{j}\right)\right]
$$

The objective of the control law is now to obtain

$$
\theta+\dot{z}=-\lambda z
$$

where $\lambda$ is a strictly positive constant, in which case

$$
\dot{V}_{2} \leq-\frac{\varepsilon_{1} \gamma}{\sigma_{b, j}}\left(\delta_{j}-\delta_{d}\right)^{2}-\kappa\left(\psi-\psi_{d}\right)^{2}-\lambda\|z\|^{2}
$$

get from (23) that

$$
\theta-\dot{\alpha}-M^{-1}\left(D \nu+g(\eta)-\tau-J^{T}(\psi) b\right)=-\lambda z,
$$

so we obtain

$$
\tau_{\mathrm{BSP}}=-M(\lambda z+\theta-\dot{\alpha})+D \nu+g(\eta)-J^{T}(\psi) b .
$$

Substituting for $z, \theta$, and $\dot{\alpha}$ in (26) yields (13). Thus, $\|\left(\delta_{j}-\right.$ $\left.\delta_{d}, \psi-\psi_{d}, z\right) \| \rightarrow 0$ exponentially fast. It follows from (17) that $\|\alpha\| \rightarrow 0$ exponentially fast and, from (19), that $\|\nu\| \rightarrow 0$ exponentially fast.

Considering the economical fuel consumption, the constraint function is introduced to the controllers and then the output of the controllers is as follows:

$$
\overline{\boldsymbol{\tau}}=E\left(\delta_{j}\right) \tau_{\mathrm{BSP}}
$$

where

$$
E\left(\delta_{j}\right)=\left[\begin{array}{ccc}
e\left(\delta_{j}\right) & 0 & 0 \\
0 & e\left(\delta_{j}\right) & 0 \\
0 & 0 & 1
\end{array}\right]
$$

and $e\left(\delta_{j}\right)$ is a proper second-order polynomial satisfying

$$
\begin{aligned}
& e\left(\delta_{j}\right) \\
& \quad= \begin{cases}0 & \left(\delta_{j} \geq \delta_{\max }\right) \\
\frac{\delta_{j}^{2}}{\Delta \delta^{2}}-2 \frac{\delta_{\max }}{\Delta \delta^{2}} \delta_{j}+\frac{\delta_{\max }^{2}}{\Delta \delta^{2}} & \left(\delta_{\min }<\delta_{j}<\delta_{\max }\right) \\
1 & \left(\delta_{j} \leq \delta_{\min }\right),\end{cases}
\end{aligned}
$$

where

$$
\Delta \delta=\delta_{\max }-\delta_{\min }
$$

and $\delta_{\max }$ and $\delta_{\min }$ are just the variables to decide the active region of the controllers. When $\delta_{j}>\delta_{\max }$, the thrusters do 


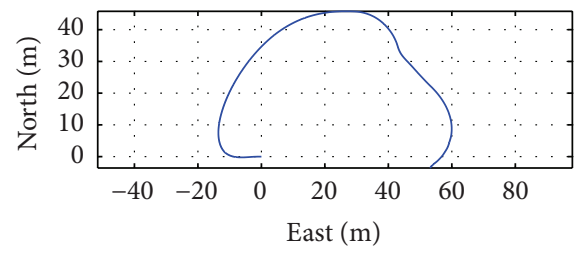

(a)

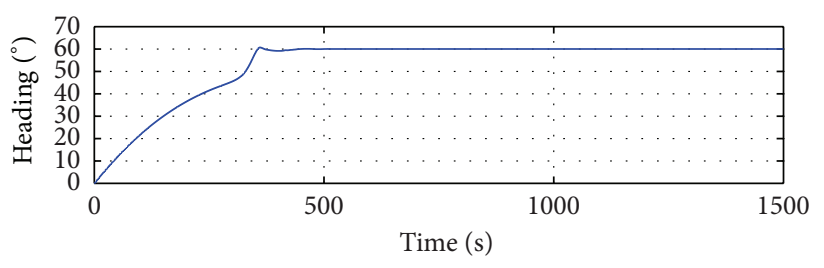

(b)

FIGURE 4: Trajectory tracking and heading of FPSO.

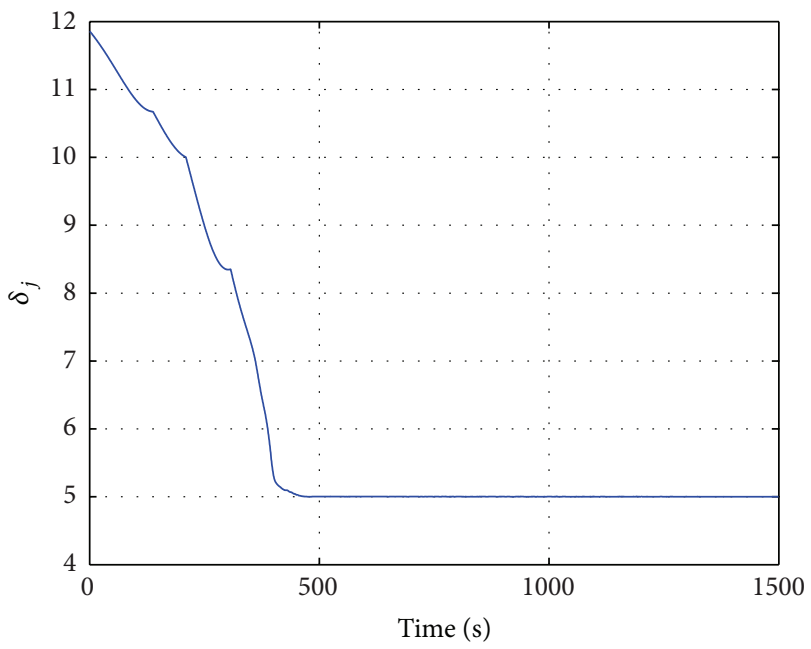

Figure 5: Minimum reliability index of mooring lines.

not work; when $\delta_{\min }<\delta_{j}<\delta_{\max }$, the thrusters work in a low load state; when $\delta_{j}<\delta_{\min }$, the thrusters work in a high load state.

\section{Simulations}

The performance of the designed controllers is validated through simulations for a certain single-point turret-moored FPSO in extreme conditions. The basic parameters of the FPSO and the data of mooring lines are shown in Table 1.

The simulation time is 3000 seconds; $\psi_{d}$ is set to $60^{\circ}$; $\delta_{s}=3.3, \delta_{\max }=9.5, \delta_{\min }=6, \delta_{d}=5$; the setting wind speed is $25 \mathrm{~m} / \mathrm{s}$ and the absolute wind direction is $45^{\circ}$; the significant wave height $H_{s}=6.3 \mathrm{~m}$; the period is $10.1 \mathrm{~s}$; the current velocity is $2.0 \mathrm{~m} / \mathrm{s}$; and the current direction is $60^{\circ}$.

The dynamic positioning simulations of the moored inertial turret-moored FPSO are carried out based on above settings and the results are shown in Figures 4, 5, and 6.

From Figure 4, we can see that the maximum position deviation of FPSO is about 50 meters, and the position is finally stabilized in the range of 10 meters. The heading is eventually stabilized at about 60 degrees. It is shown in Figure 5 that the minimum reliability index of mooring lines is stabilized as expected in extreme seas, which make sure the mooring lines are safe. From Figure 6, the $X$ and $Y$ control force are zero before 250 seconds due to the effects

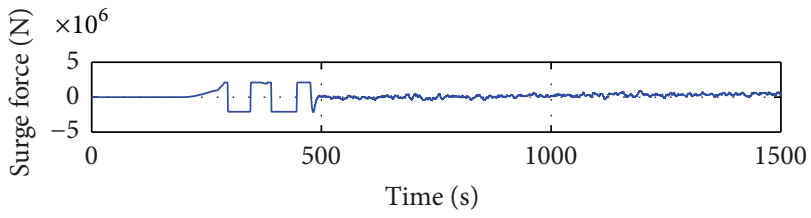

(a)

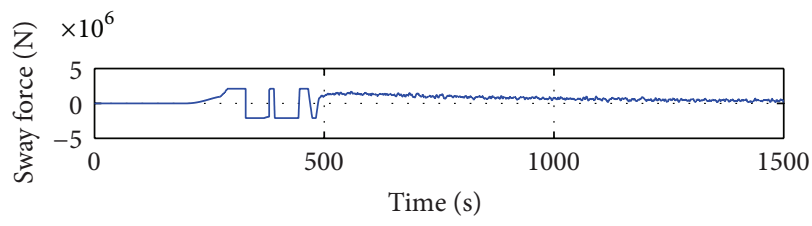

(b)

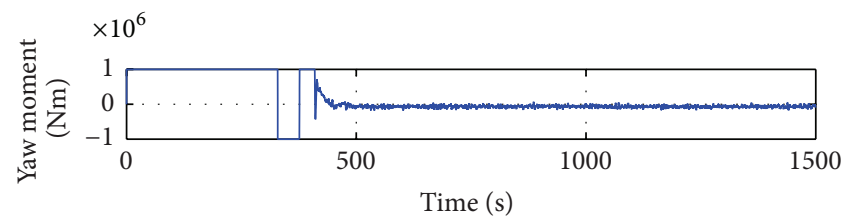

(c)

FIgURE 6: Thrust forces of the FPSO.

of constraint function, while the control forces are reduced as the reliability index up to the minimum after 500 seconds.

\section{Conclusion}

Here, the kinematics and dynamics of the internal turretmoored FPSO mathematical models were established. Structural reliability based state feedback backstepping controllers were proposed with application to the internal turret-moored FPSO model. In order to ensure the safety of all the mooring lines, the structural reliability index is selected a little larger than the critical reliability index. Finally, a series of simulation tests were carried out for a certain turret-moored FPSO with eight mooring lines. The simulations showed that the designed controllers were effective not only to keep the moored FPSO in the desired position well, but also to keep the reliability index of all mooring lines within safe ranges in extreme seas. However, the way to introduce the structural reliability index is limited to the designed backstepping controllers, which cannot be used in other controllers like PID, PI, and so on. Thus, the comparisons of control results with different controllers are not available. In the further 
TABLE 1: The data of the FPSO and mooring lines.

\begin{tabular}{lc}
\hline Name & Value \\
\hline Ship length & $310.0 \mathrm{~m}$ \\
Waterline length & $296.0 \mathrm{~m}$ \\
Ship width & $47.2 \mathrm{~m}$ \\
Draft & $18.9 \mathrm{~m}$ \\
Water depth & $500 \mathrm{~m}$ \\
Mooring line length & $1000 \mathrm{~m}$ \\
Elastic modulus & $1.868 * 108 \mathrm{~N}$ \\
Displacement & $240869 \mathrm{t}$ \\
Metacentric height & $6.6 \mathrm{~m}$ \\
Waterline coefficient & 0.9164 \\
Block coefficient & 0.85 \\
Mooring line number & 8 \\
Unit weight & $1088.6 \mathrm{~N} / \mathrm{m}$ \\
Breaking strength & $3500 \mathrm{KN}$ \\
\hline
\end{tabular}

research, a lot more attention should be paid to more types of structural reliability based controller designs.

\section{Conflict of Interests}

The authors declare that there is no conflict of interests regarding the publication of this paper.

\section{Acknowledgments}

This work is supported by the National Natural Science Foundation of China (no. 51209056), the Fundamental Research Funds for the Central Universities (no. HEUCF041401), and National Science and Technology Major Project (no. 2011ZX05027-002).

\section{References}

[1] J. P. Strand, A. J. Sørensen, and T. I. Fossen, "Design of automatic thruster assisted position mooring systems for ships," Modeling, Identification and Control, vol. 19, no. 2, pp. 61-75, 1998.

[2] D. T. Nguyen and A. J. Sørensen, "Setpoint chasing for thrusterassisted position mooring," IEEE Journal of Oceanic Engineering, vol. 34, no. 4, pp. 548-558, 2009.

[3] D. T. Nguyen and A. J. Sørensen, "Switching control for thruster-assisted position mooring," Control Engineering Practice, vol. 17, no. 9, pp. 985-994, 2009.

[4] P. I. B. Berntsen, O. M. Aamo, and B. J. Leira, "Dynamic positioning of moored vessels based on structural reliability," in Proceedings of the 45th IEEE Conference on Decision and Control (CDC '06), pp. 5906-5911, December 2006.

[5] P. Berntsen, Structural reliability based position mooring [Ph.D. dissertation], Department of Marine Technology, Norwegian University of Science and Technology, 2008.

[6] S. Fang and M. Blanke, "Fault monitoring and fault recovery control for position-moored vessels," International Journal of Applied Mathematics and Computer Science, vol. 21, no. 3, pp. 467-478, 2011.
[7] S. Fang, B. J. Leira, and M. Blanke, "Position mooring control based on a structural reliability criterion," Structural Safety, vol. 41, pp. 97-106, 2013.

[8] T. Fossen, Marine Control Systems, Marine Cybernetics, Trondheim, Norway, 2002.

[9] C. Liu, The Calculation and Analysis of Mooring System, Wuhan University of Technology, 2007.

[10] T. Fossen, Handbook of Marine Craft Hydrodynamics and Motion Control, John Wiley \& Sons, 2011.

[11] M. Jian, Simulation of Ship Manoeuvring Motion with Six Degrees of Freedom in Waves, Harbin Engineering University, 2009.

[12] X. Jia and Y. Yang, Mathematical Model of Ship Motion, Dalian Maritime University publication, 1999.

[13] H. Madsen, S. Krenk, and N. Lind, Methods of Structural Safety, Prentice-Hall, New York, NY, USA, 1986. 


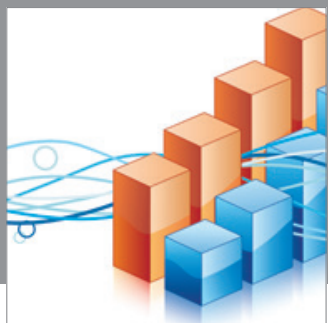

Advances in

Operations Research

mansans

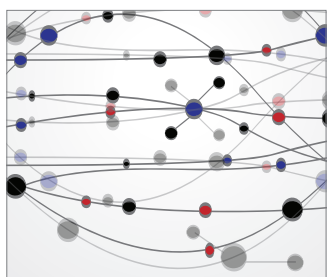

The Scientific World Journal
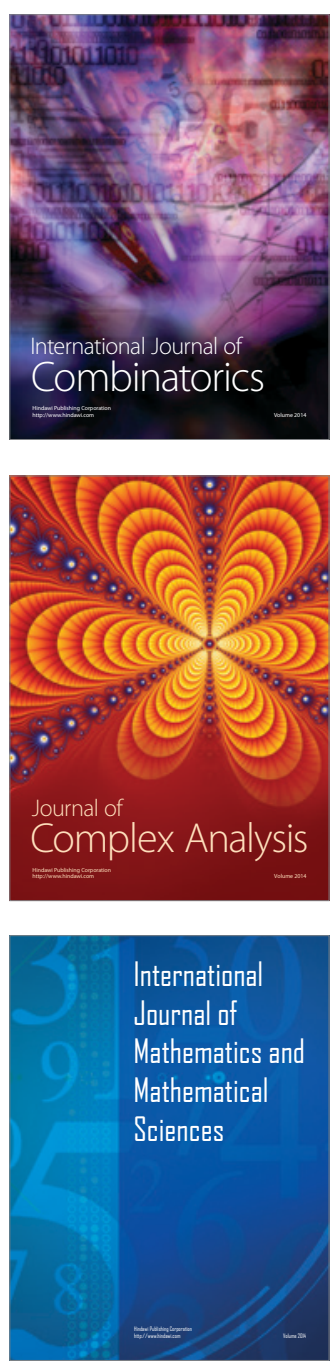
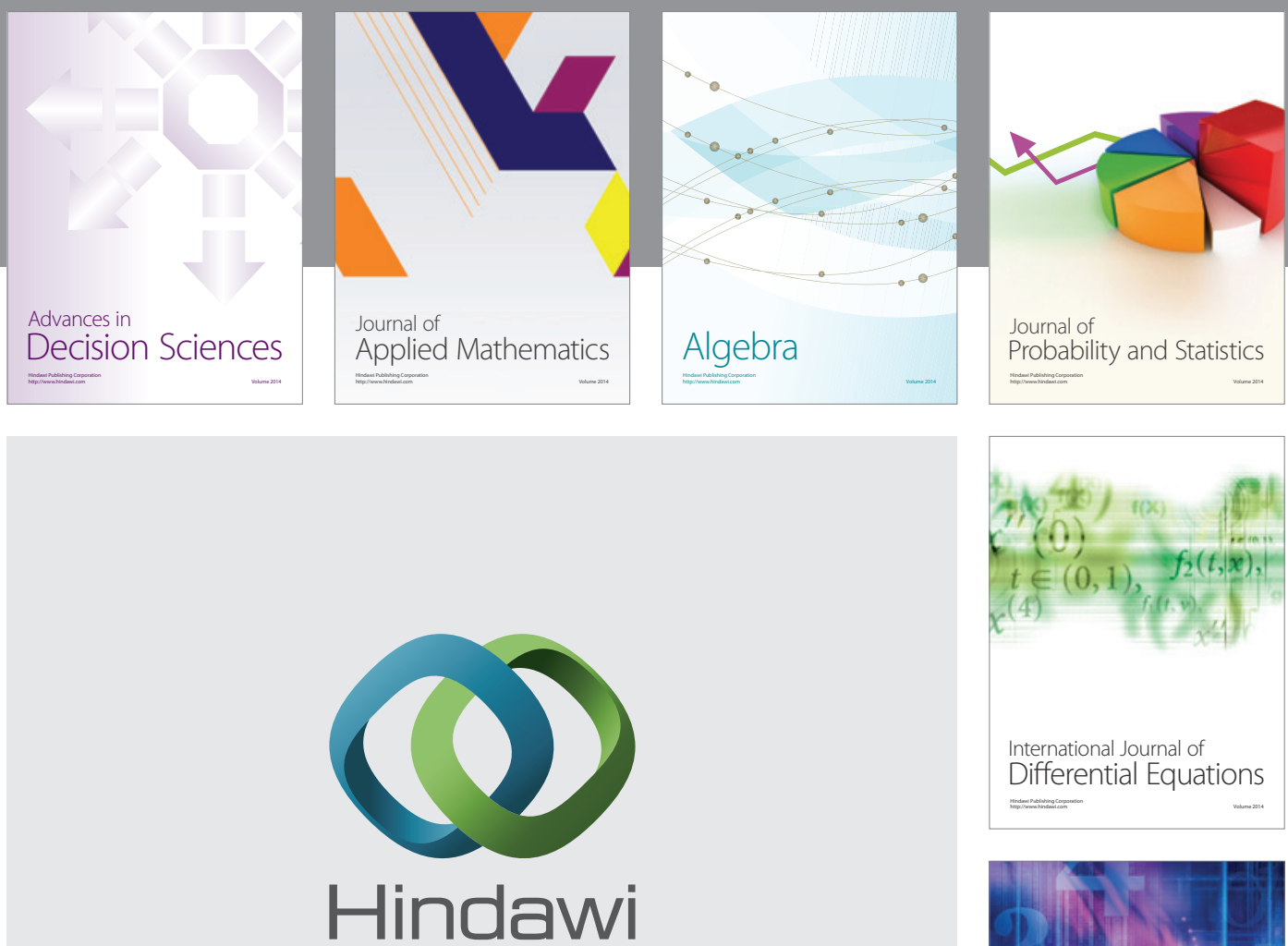

Submit your manuscripts at http://www.hindawi.com
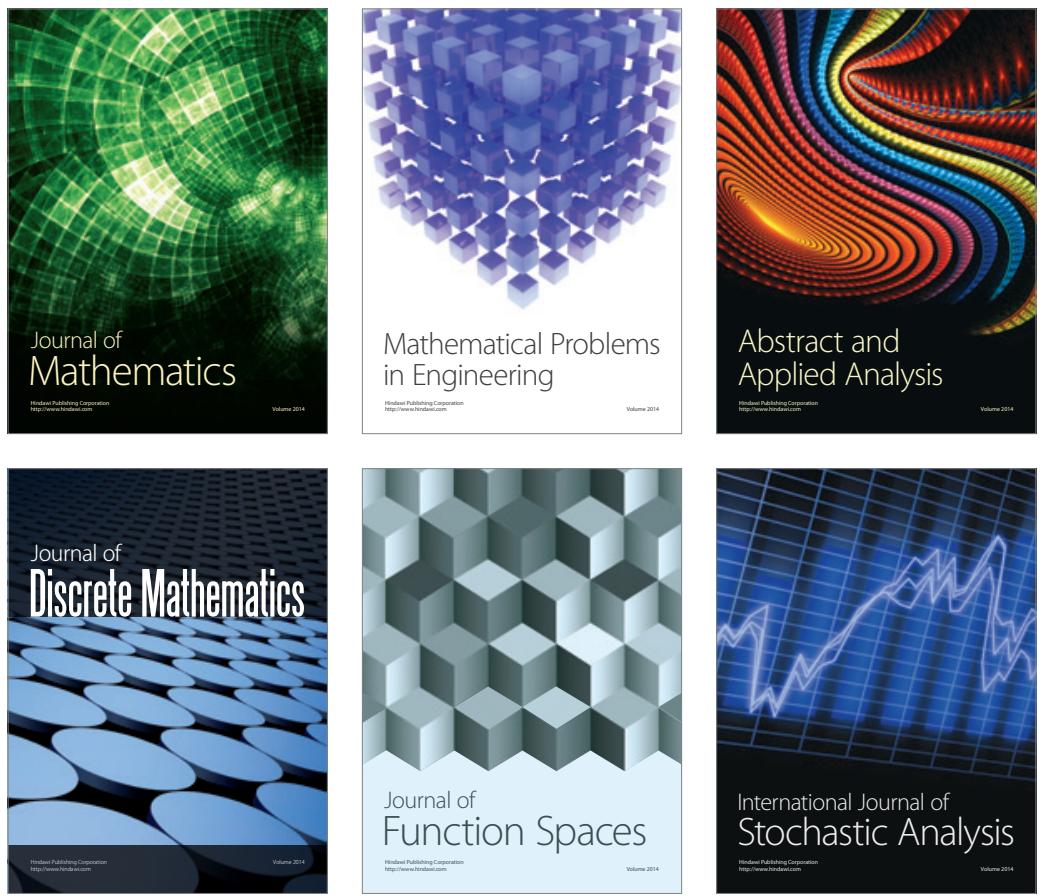

Journal of

Function Spaces

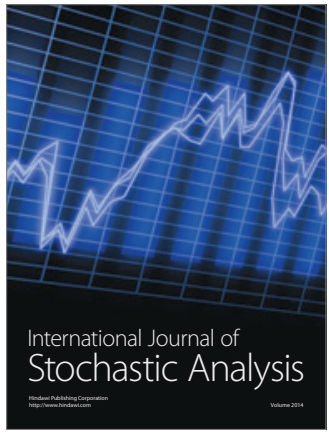

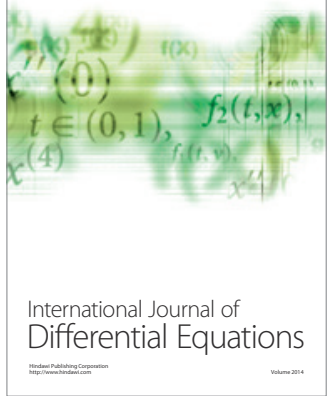
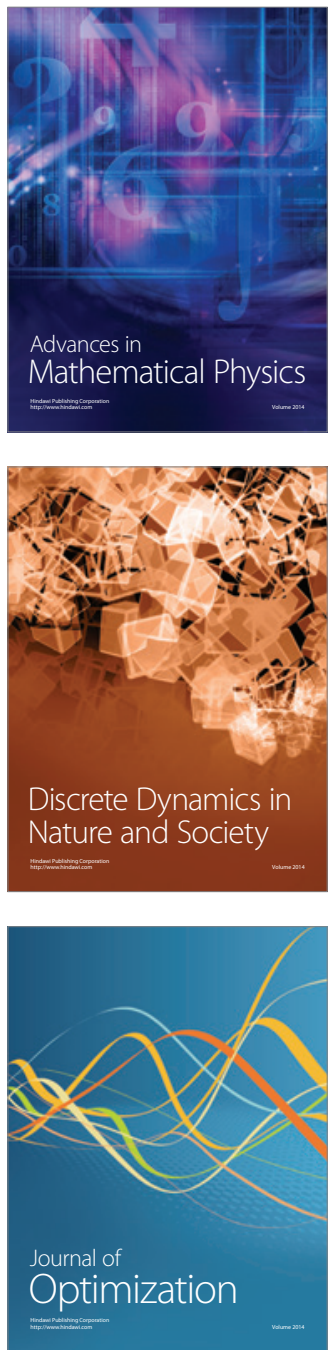